\title{
Obesidade geral e abdominal em idosos do Sul do Brasil: resultados do estudo COMO VAI?
}

\author{
General and abdominal obesity among the elderly \\ from Southern Brazil: results of the HOW ARE YOU DOING? \\ (COMO VAI?) study
}

${ }^{1}$ Programa de PósGraduação em Epidemiologia, Universidade Federal de Pelotas. R. Marechal Deodoro $1160 / 3^{\circ}$ andar, Centro. 96020-220

Pelotas RS Brasil. carolinercosta@gmail.com

\begin{abstract}
This is a cross-sectional population-based study aimed to measure the prevalence and to identify associated factors with the occurrence of general and abdominal obesity among the elderly living in the urban area of Pelotas, State of Rio Grande do Sul. The process of cluster sampling was conducted in two stages. Overall obesity was defined by Body Mass Index (BMI) from measurements of weight and height estimated by knee height. Obesity was considered when BMI was $\geq$ $30 \mathrm{~kg} / \mathrm{m}^{2}$. Abdominal obesity was evaluated by waist circumference, being considered obese when it was $>102 \mathrm{~cm}$ for men and $>88 \mathrm{~cm}$ for women. The crude and adjusted analyses were performed using Poisson regression. The prevalence of obesity was 29.9\% (CI95\%: 27.5-32.4) and abdominal obesity, 50.4\% (CI95\%: 47.8-53.1). After adjustment, the prevalence of both outcomes were higher in women, non-smokers, hypertensives and diabetics and lower in the physically active in their leisure time. Age was inversely associated with general and abdominal obesity. Reducing obesity in this population depends on the planning of health policies actively encourage physical activity, keeping in mind the limitations and health conditions of the elderly.
\end{abstract}

Key words Obesity, Abdominal obesity, Elderly, Prevalence, Anthropometry
Resumo Estudo transversal de base populacional com objetivo de medir as prevalências e identificar os fatores associados à ocorrência de obesidade geral e abdominal entre idosos residentes na área urbana de Pelotas, RS. O processo de amostragem por conglomerados foi realizado em dois estágios. A obesidade geral foi definida pelo Índice de Massa Corporal (IMC), a partir das medidas de peso e altura estimada através da altura do joelho. Considerou-se como obeso quando IMC $\geq 30 \mathrm{~kg} / \mathrm{m}^{2}$. A obesidade abdominal foi avaliada pela circunferência da cintura, sendo considerado obeso quando $>102 \mathrm{~cm}$ para homens $e>88 \mathrm{~cm}$ para mulheres. As análises brutas e ajustadas foram realizadas por meio da regressão de Poisson. A prevalência de obesidade geral foi 29,9\% (IC95\%: 27,5-32,4) e de abdominal 50,4\% (IC95\%: 47,8-53,1). Após o ajuste, as prevalências de ambos os desfechos foram maiores em mulheres, não fumantes, hipertensose diabéticos e menores em fisicamente ativos no lazer. Idade apresentou associação inversa com obesidade geral e abdominal. A redução da obesidade nesta população depende do planejamento de políticas de saúde que englobem o incentivo à prática de atividade física, considerando as limitações e as condições de saúde do idoso.

Palavras-chave Obesidade, Obesidade abdominal, Idoso, Prevalência, Antropometria 


\section{Introdução}

O crescimento vertiginoso da população idosa (60 anos ou mais ${ }^{1}$ ) traz, de imediato, aumento da carga de doenças crônicas não transmissíveis (DCNT), típicas dessa faixa etária, com destaque para as cardiovasculares, hipertensão arterial sistêmica (HAS) e diabetes mellitus (DM) ${ }^{2}$. Além de poder afetar a qualidade de vida do idoso, por comprometer a sua funcionalidade, dificultando ou impedindo o desempenho das atividades cotidianas de forma independente ${ }^{2}$, a presença dessas doenças requer cuidado continuado de média $\mathrm{e}$ alta complexidade, o que eleva o custo da prestação de serviços em saúde ${ }^{3}$.

A obesidade é um dos principais fatores associados às DCNT. Sua ocorrência é de caráter multifatorial, podendo ser resultado da combinação de fatores genéticos e fisiológicos com um ambiente obesogênico, caracterizado, principalmente, pela presença de atividade física insuficiente e de hábitos alimentares inadequados ${ }^{4}$. Em nível mundial, a prevalência de obesidade praticamente dobrou entre 1980 e 2008, passando de $5 \%$ em homens e $8 \%$ em mulheres para $10 \%$ e $14 \%$, respectivamente ${ }^{5}$. No Brasil, a prevalência de obesidade em 2008 foi de $13 \%$ em homens e $17 \%$ em mulheres 6 .

Entre os idosos, as alterações fisiológicas decorrentes do processo de envelhecimento, como, por exemplo, o declínio da altura (aproximadamente $2-4 \mathrm{~cm}$ ao longo da vida, comparado à altura máxima ${ }^{7}$ ), causado por compressão vertebral e perda do tônus muscular, a redução da massa muscular devido à sua transformação em gordura intramuscular e, principalmente, a modificação na quantidade e distribuição do tecido adiposo subcutâneo, com acúmulo na região abdominal ${ }^{8}$, representam diferenciações entre adultos e idosos e, por esse motivo, devem ser consideradas na definição de obesidade ${ }^{2}$.

A obesidade, em qualquer de suas formas de manifestação, aumenta o risco para doenças cardiovasculares, DM, doenças musculoesqueléticas e alguns tipos de câncer9. Além disso, o acúmulo de gordura na região abdominal altera o perfil metabólico, com diminuição da tolerância à glicose, redução da sensibilidade à insulina e perfis lipídicos adversos ${ }^{10}$. Apesar de toda esta importância, no contexto brasileiro são escassos os estudos de base populacional abordando este tema na população idosa.

Este estudo teve por objetivo medir as prevalências e identificar os fatores associados à ocorrência de obesidade geral e abdominal na popu- lação idosa residente na área urbana do município de Pelotas, RS.

\section{Métodos}

O presente estudo foi conduzido em Pelotas, município de médio porte, localizado na chamada Metade do Sul do Rio Grande do Sul, que tem como base econômica o agronegócio e o comércio. É considerado o terceiro município mais populoso do estado, com cerca de 330 mil habitantes $(93,3 \%$ urbana), sendo, aproximadamente, $15 \%$ indivíduos idosos ${ }^{11}$.

Este estudo faz parte de um inquérito maior que investiga a situação de saúde da população pelotense desde 1999, sob forma de consórcio, realizado por mestrandos em epidemiologia a cada dois $\operatorname{anos}^{12}$. Trata-se de um estudo transversal de base populacional sobre a população idosa (60 anos ou mais) residente na área urbana do município. Em 2014, o estudo foi conhecido como Consórcio de Mestrado Orientado para a Valorização da Atenção ao Idoso (COMO VAI?) e a coleta de dados foi realizada entre os meses de janeiro e agosto.

O cálculo do tamanho amostral para o estudo de prevalência considerou nível de confiança de $95 \%$, efeito de delineamento de 1,3 e prevalência de $33,5 \%$ para a obesidade geral e $45 \%$ para a abdominal ${ }^{13}$. O erro máximo aceitável foi de quatro pontos percentuais. Com base nesses parâmetros amostrais, este estudo deveria incluir pelo menos 916 idosos. Destaca-se que esse valor está acrescido de 20\% para a ocorrência de eventuais perdas.

Para o estudo de associação, os cálculos de tamanho amostral foram realizados a posteriori. Considerou-se poder de $80 \%$, nível de confiança de $95 \%$, razões de prevalências variando entre 1,25 e 1,90 e frequências de exposição de $23 \%$ e $81 \%$. Além disso, acrescentou-se $20 \%$ para possíveis perdas e recusas e $15 \%$ para controle de fatores de confusão. Com base nesses parâmetros, seriam necessários pelo menos 1.264 idosos. Para algumas variáveis contidas no modelo hierárquico de análise (cor da pele, escolaridade, classe econômica, situação conjugal, doença cardiovascular e hipercolesterolemia) não foi possível trabalhar com esses parâmetros amostrais, em virtude de requerer um tamanho amostral substancialmente maior que o passível de obtenção neste estudo. Apesar disso, essas variáveis foram mantidas na análise em função da definição prévia do modelo hierárquico.

O processo de amostragem por conglomerados foi realizado em dois estágios, com base 
nas informações do Censo Demográfico de 2010 do Instituto Brasileiro de Geografia e Estatística $(\text { IBGE })^{11}$. Primeiramente, todos os 488 setores censitários da zona urbana do município foram listados em ordem crescente, de acordo com a renda média, garantindo a participação de indivíduos com diferentes situações socioeconômicas. Setores com menos que 15 idosos foram agrupados, resultando em 469 setores para o sorteio sistemático.

Considerando haver 0,43 idosos por domicilio, definiu-se que seriam selecionados 31 domicílios por setor, possibilitando a identificação de, no mínimo, 12 idosos. Para atingir o tamanho amostral da pesquisa como um todo foram sorteados 133 setores censitários, a partir de um "pulo" sistemático. Os domicílios permanentes ocupados, dos setores selecionados, foram listados e sorteados sistematicamente e todos os indivíduos idosos, moradores dos domicílios sorteados, foram incluídos na amostra. Para este estudo, foram excluídos todos aqueles que se mostraram impossibilitados de permanecer em posição ereta, bem como os que utilizavam prótese ou gesso ou amputados de membros inferiores.

A obesidade geral foi investigada através do Índice de Massa Corporal (IMC) e utilizou-se o ponto de corte preconizado pela Organização Mundial da Saúde (OMS) ${ }^{14}$, que classifica como obesos indivíduos com IMC $\geq 30 \mathrm{~kg} / \mathrm{m}^{2}$. Para o cálculo do IMC foram utilizadas medidas de peso e altura, esta estimada através da altura do joelho, por ser mais adequada para a população-alvo ${ }^{14}$, sendo empregadas as equações preditivas propostas por Chumlea e $\mathrm{Guo}^{15}$.

A circunferência da cintura foi utilizada para avaliar a obesidade abdominal, medida com uma fita métrica não extensível diretamente sobre a pele na região mais estreita do tronco, entre o tórax e o quadril, sendo a leitura feita no momento da expiração. Somente em caso de não haver ponto mais estreito, a medida era feita no ponto médio entre a última costela e a crista ilíaca. Esta técnica é recomendada pela $\mathrm{OMS}^{14}$ e pelo $\mathrm{Mi}$ nistério da Saúde ${ }^{16}$, sendo utilizada por diversos estudos ${ }^{13,17,18}$. Foram classificados como portadores de obesidade abdominal os indivíduos com circunferência da cintura $>102 \mathrm{~cm}$ para homens e $>88 \mathrm{~cm}_{\text {para mulheres }}{ }^{14}$.

Para identificar os fatores associados à obesidade geral e abdominal foram utilizadas as seguintes variáveis independentes: idade (obtida em anos completos e agrupada em três categorias: 60-69; 70-79; 80 anos ou mais), sexo, cor da pele (observada pela entrevistadora e categorizada como branca ou preta/parda/amarela/indígena), escolaridade (agrupada em quatro categorias: 0-3 anos de estudo; 4-7; 8-11; 12 ou mais), classe econômica (classificação da Associação Brasileira de Empresas de Pesquisa - $\mathrm{ABEP}^{19}$, agrupada em três categorias: classe A/B-mais ricos, C ou D/E-mais pobres) e situação conjugal (com ou sem companheiro/a).

Foram ainda incluídas como variáveis independentes tabagismo atual (não ou sim, considerando como fumante aquele indivíduo que fumou todos os dias, pelo menos uma vez, durante 30 dias) e atividade física de lazer, coletada através do questionário IPAQ - International Physical Activity Questionnaire ${ }^{20}$ (insuficientemente ativo: $<150$ minutos/semana; ativo: $\geq 150$ minutos/ semana), bem como a ausência ou presença autorreferida de HAS, DM, doença cardiovascular e hipercolesterolemia. As variáveis relacionadas à saúde foram coletadas através da pergunta Algum médico ou profissional de saúde já disse que o(a) sr.(a) tem hipertensão (pressão alta), mesmo que controlada; diabetes; problema do coração, atual ou antigo; colesterol alto ou gordura no sangue?

A coleta dos dados foi realizada por entrevistadoras com, no mínimo, ensino médio completo, previamente treinadas e padronizadas para a coleta das medidas antropométricas, de acordo com os critérios propostos por Habicht ${ }^{21}$. O questionário utilizado foi testado pelos autores anteriormente ao início da pesquisa a partir de estudo piloto. Em quase toda a coleta de dados, o estudo contou com auxílio de um veículo para transporte das entrevistadoras até os domicílios selecionados.

A entrada dos dados se deu por meio de netbooks da marca Samsung ${ }^{\circledR}$ modelo N150 plus, e do software Pendragon ${ }^{\circledR}$. Para a coleta das medidas antropométricas, foram utilizados os seguintes instrumentos: balanças eletrônicas da marca Tanita ${ }^{\circledR}$, modelo UM-080, com capacidade máxima de 150 quilogramas e precisão de 100 gramas, para obtenção do peso; antropômetro infantil em madeira da marca Indaiá ${ }^{\circledR}$, com escala de 100 centímetros e graduação em milímetros, para medida de altura do joelho; e fitas métricas não extensíveis da marca Cescorf ${ }^{\circledR}$, com extensão de 2 metros e graduação em milímetros, para medida de circunferência da cintura. As técnicas de medição seguiram as recomendações da $\mathrm{OMS}^{11}$.

O controle de qualidade foi feito através de nova visita e entrevista com uma versão reduzida do questionário original a $10 \%$ dos entrevistados, esses selecionados aleatoriamente. Para este estudo, a questão utilizada foi: Alguém pesou o(a) $s r(a)$ ? Foi testada a concordância esperada além 
do acaso entre as respostas utilizando-se o teste Kappa, o qual resultou em $87 \%$ de repetibilidade.

As análises estatísticas foram realizadas por meio do programa estatístico Stata, versão 12.1 (Stata Corporation, College Station, USA). O efeito de amostragem por conglomerados foi considerado em todas as análises, através do comando survey (svy). A significância estatística de cada variável foi avaliada através dos testes de Wald de heterogeneidade ou de tendência linear. Foram realizadas análises brutas e ajustadas através da regressão de Poisson. Testes de interação entre os desfechos (obesidade geral e abdominal), sexo e outras variáveis independentes foram realizados, obtendo-se resultado não significativo, entretanto realizou-se estratificação das análises por sexo por ser considerada uma informação importante ao tema.

Para a análise multivariável, foi construído um modelo conceitual hierárquico com as variáveis demográficas e socioeconômicas encontradas no primeiro nível, as comportamentais no segundo e as relacionadas à saúde no mais proximal para cada desfecho ${ }^{22}$. Utilizou-se o procedimento de seleção para trás (backward) por níveis, incluindo somente as variáveis com $\mathrm{p}<0,2$ na análise bruta. De acordo com este método, as variáveis do primeiro nível foram ajustadas entre si, sendo mantidas no modelo aquelas que apresentaram valor $-\mathrm{p}<0,05$ na análise ajustada. Após, foram incluídas as variáveis comportamentais, ajustando-as para as variáveis do primeiro nível que permaneceram no modelo, e para as do segundo com $\mathrm{p}<0,05$. Por fim, as variáveis relacionadas à morbidade autorreferida foram incluídas na análise, sendo ajustadas para as variáveis dos dois níveis anteriores, bem como para as do terceiro nível com $p<0,05$. Foram considerados fatores associados ao desfecho aquelas variáveis com $\mathrm{p}<0,05$.

Este estudo foi aprovado pelo Comitê de Ética em Pesquisa da Faculdade de Medicina da Universidade Federal de Pelotas. A utilização de um número para cada participante no banco de dados garantiu a confidencialidade dos dados pessoais. Anteriormente à entrevista, foi obtida assinatura no termo de consentimento livre e esclarecido de todos os participantes, concordando com a participação na pesquisa.

\section{Resultados}

O presente estudo identificou 1.844 idosos. Destes, foi possível entrevistar 1.451, corresponden- do a uma taxa de resposta de $78,7 \%$. Dentre as perdas e recusas, $59,3 \%$ eram mulheres, semelhante à amostra $(\mathrm{p}=0,198)$, e a média de idade foi 69,5 anos (DP $\pm 8,6$ ), com diferença em relação à amostra $(70,7 ; \mathrm{DP} \pm 8,2 ; \mathrm{p}=0,011)$. Entre os idosos entrevistados, 87 não tinham informação de peso ou altura do joelho e 78 de circunferência da cintura, por estarem entre os critérios de exclusão. $\mathrm{O}$ efeito de delineamento amostral do estudo foi 1,11 para obesidade geral e 1,06 para obesidade abdominal.

A Tabela 1 mostra que 52\% dos idosos possuíam entre 60 e 69 anos de idade, $63 \%$ pertenciam ao sexo feminino, $84 \%$ eram de cor da pele branca, $53 \%$ pertenciam ao estrato $\mathrm{C}$ de classe econômica, $37 \%$ possuíam quatro anos ou mais de escolaridade e $53 \%$ viviam com companheiro. Com relação às variáveis comportamentais e de morbidade, a grande maioria dos idosos relatou não fumar atualmente $(87,4 \%)$, ser insuficientemente ativa fisicamente $(81,4 \%)$, enquanto $67 \%, 24 \%, 32 \%$ e $41 \%$ referiu ter HAS, DM, doença cardiovascular e hipercolesterolemia, respectivamente.

Entre os idosos estudados, 29,9\% (IC95\%: 27,5-32,4) foram considerados obesos e cerca de metade da amostra [50,4\% (IC95\%: 47,8-53,1)] apresentou obesidade abdominal (Tabela 1). As prevalências de obesidade geral e abdominal foram significativamente maiores entre as mulheres (Figura 1). Observou-se diminuição progressiva da obesidade geral com o aumento da idade e foi encontrada maior prevalência em indivíduos com HAS, DM e doença cardiovascular autorreferidas. Indivíduos que referiram fumar e aqueles fisicamente ativos apresentaram menores prevalências de obesidade (Tabela 2). Para obesidade abdominal, maiores prevalências foram observadas em idosos que viviam sem companheiro e naqueles que referiram ter HAS, DM, doença cardiovascular ou hipercolesterolemia. Observaram-se prevalências menores na faixa mais avançada de idade ( 80 anos ou mais) e em idosos fisicamente ativos e que referiram fumar atualmente (Tabela 2).

$\mathrm{Na}$ análise ajustada, realizada a partir de modelo conceitual hierárquico, as variáveis que permaneceram associadas à obesidade geral e à obesidade abdominal (Tabela 2) foram idade, sexo, tabagismo atual, atividade física no lazer, HAS e DM.

Para obesidade geral, as mulheres apresentaram prevalência 35\% maior, quando comparadas aos homens. Observou-se uma relação inversa entre idade e obesidade: idosos com 70 a 79 anos tiveram prevalência $24 \%$ menor, comparandose aos de 60 a 69 anos, e aqueles com 80 anos 
Tabela 1. Descrição da amostra em termos de variáveis demográficas, socioeconômicas, comportamentais e de morbidade $(\mathrm{n}=1.451)$. Pelotas, RS, Brasil, 2014.

\begin{tabular}{|c|c|c|c|}
\hline Variáveis & $\begin{array}{c}\text { Homens } \\
\text { n (\%) }\end{array}$ & $\begin{array}{c}\text { Mulheres } \\
\text { n (\%) }\end{array}$ & $\begin{array}{l}\text { Total } \\
\mathbf{n}(\%)\end{array}$ \\
\hline \multicolumn{4}{|l|}{ Idade $($ anos $)(\mathrm{n}=1.446)$} \\
\hline $60-69$ & $289(53,9)$ & $467(51,3)$ & $756(52,3)$ \\
\hline $70-79$ & $172(32,1)$ & $288(31,7)$ & $460(31,8)$ \\
\hline $80+$ & $75(14,0)$ & $155(17,0)$ & $230(15,9)$ \\
\hline \multicolumn{4}{|l|}{ Cor da pele $(\mathrm{n}=1.447)$} \\
\hline Branca & $454(84,7)$ & $757(83,1)$ & $1.211(83,7)$ \\
\hline Preta/Parda/Amarela/Indígena & $82(15,3)$ & $154(16,9)$ & $236(16,3)$ \\
\hline \multicolumn{4}{|l|}{ Escolaridade (anos de estudo) $(\mathrm{n}=1.437)$} \\
\hline $0-3$ & $205(38,5)$ & $328(36,3)$ & $533(37,0)$ \\
\hline $4-7$ & $169(31,7)$ & $276(30,5)$ & $445(31,0)$ \\
\hline $8-11$ & $55(10,3)$ & $88(9,7)$ & $143(10,0)$ \\
\hline $12+$ & $104(19,5)$ & $212(23,5)$ & $316(22,0)$ \\
\hline \multicolumn{4}{|l|}{ Classe econômica (ABEP) $(\mathrm{n}=1.372)$} \\
\hline A/B (mais ricos) & $200(39,4)$ & $283(32,8)$ & $483(35,2)$ \\
\hline $\mathrm{C}$ & $248(48,8)$ & $472(54,6)$ & $720(52,5)$ \\
\hline $\mathrm{D} / \mathrm{E}$ (mais pobres) & $60(11,8)$ & $109(12,6)$ & $169(12,3)$ \\
\hline \multicolumn{4}{|l|}{ Situação conjugal $(\mathrm{n}=1.447)$} \\
\hline Com companheiro (a) & $408(76,1)$ & $355(39,0)$ & $763(52,7)$ \\
\hline Sem companheiro (a) & $128(23,9)$ & $556(61,0)$ & $684(47,3)$ \\
\hline Tabagismo atual $(\mathrm{n}=1.446)$ & $76(14,2)$ & $106(11,7)$ & $182(12,6)$ \\
\hline \multicolumn{4}{|l|}{ Atividade física no lazer $(\mathrm{n}=1.391)$} \\
\hline Insuficientemente ativo & $391(76,4)$ & $742(84,4)$ & $1.133(81,4)$ \\
\hline Ativo & $121(23,6)$ & $137(15,6)$ & $258(18,6)$ \\
\hline Hipertensão arterial sistêmica $(\mathrm{n}=1.447)$ & $340(63,5)$ & $625(68,5)$ & $965(66,7)$ \\
\hline Diabetes $(\mathrm{n}=1.447)$ & $129(24,1)$ & $211(23,1)$ & $340(23,5)$ \\
\hline Doença cardiovascular $(\mathrm{n}=1.446)$ & $174(32,5)$ & $291(31,9)$ & $465(32,2)$ \\
\hline Hipercolesterolemia $(\mathrm{n}=1.446)$ & $176(33,0)$ & $413(45,3)$ & $589(40,7)$ \\
\hline Obesidade geral $(\mathrm{n}=1.364)$ & $120(24,0)$ & $288(33,4)$ & $408(29,9)$ \\
\hline Obesidade abdominal $(\mathrm{n}=1.373)$ & $163(32,3)$ & $529(60,9)$ & $692(50,4)$ \\
\hline Total & $537(37,0)$ & $914(63,0)$ & $1.451(100,0)$ \\
\hline
\end{tabular}

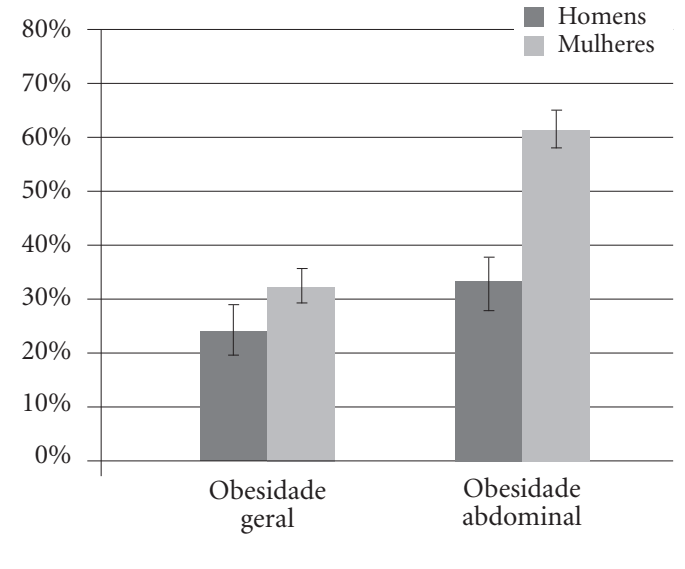

Figura 1. Prevalências e intervalos de confiança de 95\% de obesidade geral e abdominal conforme o sexo. Pelotas, RS, Brasil, 2014. ou mais apresentaram prevalência 53\% menor. Entre as variáveis comportamentais, indivíduos que referiram ser fumantes no momento da entrevista apresentaram prevalência de obesidade $29 \%$ menor do que os não fumantes e aqueles fisicamente ativos mostraram uma prevalência $26 \%$ menor, quando comparados aos insuficientemente ativos. Ainda, idosos que referiram ser hipertensos e diabéticos tiveram prevalências de obesidade $37 \%$ e $22 \%$ maiores, respectivamente, quando comparados aos que não referiram tais doenças (Tabela 3).

Em relação à obesidade abdominal, as mulheres tiveram prevalência $80 \%$ maior, quando comparadas aos homens. Assim como para obesidade geral, observou-se uma relação inversa entre idade e obesidade abdominal, sendo que idosos com idade entre 70 e 79 anos apresentaram pre- 
Tabela 2. Análises bruta e ajustada entre obesidade geral e abdominal e variáveis independentes para amostra total. Pelotas, RS, Brasil, 2014.

\begin{tabular}{|c|c|c|c|c|}
\hline \multirow[b]{2}{*}{ Variáveis } & \multicolumn{2}{|c|}{ Obesidade geral } & \multicolumn{2}{|c|}{ Obesidade abdominal } \\
\hline & $\begin{array}{c}\text { Bruta } \\
\text { RP (IC95\%) }\end{array}$ & $\begin{array}{c}\text { Ajustada } \\
\text { RP (IC95\%) }\end{array}$ & $\begin{array}{c}\text { Bruta } \\
\text { RP (IC95\%) }\end{array}$ & $\begin{array}{c}\text { Ajustada } \\
\text { RP (IC95\%) }\end{array}$ \\
\hline \multicolumn{5}{|l|}{ 10 Nível } \\
\hline Idade (anos) & $\mathrm{p}<0,001^{*}$ & $\mathrm{p}<0,001^{*}$ & $\mathrm{p}=0,028$ & $\mathrm{p}=0,001^{*}$ \\
\hline $60-69$ & 1,00 & 1,00 & 1,00 & 1,00 \\
\hline $70-79$ & $0,81(0,68 ; 0,97)$ & $0,76(0,64 ; 0,91)$ & $1,05(0,93 ; 1,18)$ & $0,98(0,88 ; 1,09)$ \\
\hline $80+$ & $0,54(0,41 ; 0,72)$ & $0,47(0,35 ; 0,63)$ & $0,82(0,70 ; 0,97)$ & $0,73(0,62 ; 0,86)$ \\
\hline Sexo & $\mathrm{p}=0,002$ & $\mathrm{p}=0,004$ & $\mathrm{p}<0,001$ & $\mathrm{p}<0,001$ \\
\hline Masculino & 1,00 & 1,00 & 1,00 & 1,00 \\
\hline Feminino & $1,39(1,13 ; 1,72)$ & $1,35(1,11 ; 1,66)$ & $1,89(1,65 ; 2,16)$ & $1,80(1,57 ; 2,06)$ \\
\hline Cor da pele & $\mathrm{p}=0,229$ & - & $\mathrm{p}=0,277$ & - \\
\hline Branca & 1,00 & - & 1,00 & - \\
\hline Preta/Parda/Amarela/Indígena & $1,14(0,92 ; 1,41)$ & - & $0,91(0,78 ; 1,08)$ & - \\
\hline Escolaridade & $\mathrm{p}=0,302$ & - & $\mathrm{p}=0,568$ & - \\
\hline $0-3$ & 1,00 & - & 1,00 & - \\
\hline $4-7$ & $1,19(0,99 ; 1,44)$ & - & $1,07(0,95 ; 1,21)$ & - \\
\hline $8-11$ & $1,08(0,81 ; 1,44)$ & - & $1,01(0,85 ; 1,20)$ & - \\
\hline $12+$ & $1,13(0,91 ; 1,41)$ & - & $0,97(0,83 ; 1,13)$ & - \\
\hline Classe econômica (ABEP) & $\mathrm{p}=0,205$ & - & $\mathrm{p}=0,103$ & $\mathrm{p}=0,205$ \\
\hline A/B (mais ricos) & 1,00 & - & 1,00 & 1,00 \\
\hline $\mathrm{C}$ & $1,02(0,84 ; 1,24)$ & - & $1,09(0,96 ; 1,25)$ & $1,05(0,92 ; 1,18)$ \\
\hline D/E (mais pobres) & $0,76(0,53 ; 1,09)$ & - & $0,91(0,74 ; 1,13)$ & $0,89(0,72 ; 1,10)$ \\
\hline Situação conjugal & $\mathrm{p}=0,448$ & - & $\mathrm{p}=0,012$ & $\mathrm{p}=0,931$ \\
\hline Com companheiro (a) & 1,00 & - & 1,00 & 1,00 \\
\hline Sem companheiro (a) & $0,94(0,93 ; 1,04)$ & - & $1,16(1,03 ; 1,31)$ & $1,01(0,89 ; 1,13)$ \\
\hline \multicolumn{5}{|l|}{ 2o Nível } \\
\hline Tabagismo atual & $\mathrm{p}=0,054$ & $\mathrm{p}=0,029$ & $\mathrm{p}=0,006$ & $\mathrm{p}=0,016$ \\
\hline Não & 1,00 & 1,00 & 1,00 & 1,00 \\
\hline Sim & $0,73(0,53 ; 1,01)$ & $0,71(0,52 ; 0,97)$ & $0,76(0,62 ; 0,92)$ & $0,79(0,66 ; 0,96)$ \\
\hline Atividade física (lazer) & $\mathrm{p}=0,034$ & $\mathrm{p}=0,022$ & $\mathrm{p}<0,001$ & $\mathrm{p}<0,001$ \\
\hline Insuficientemente ativo & 1,00 & 1,00 & 1,00 & 1,00 \\
\hline Ativo & $0,75(0,58 ; 0,98)$ & $0,74(0,57 ; 0,96)$ & $0,70(0,58 ; 0,85)$ & $0,74(0,62 ; 0,87)$ \\
\hline \multicolumn{5}{|l|}{ 3o Nível } \\
\hline Hipertensão arterial & $\mathrm{p}<0,001$ & $\mathrm{p}=0,003$ & $\mathrm{p}<0,001$ & $\mathrm{p}<0,001$ \\
\hline Não & 1,00 & 1,00 & 1,00 & 1,00 \\
\hline Sim & $1,49(1,22 ; 1,82)$ & $1,37(1,12 ; 1,68)$ & $1,57(1,37 ; 1,80)$ & $1,44(1,25 ; 1,65)$ \\
\hline Diabetes & $\mathrm{p}=0,002$ & $\mathrm{p}=0,025$ & $\mathrm{p}<0,001$ & $\mathrm{p}=0,011$ \\
\hline Não & 1,00 & 1,00 & 1,00 & 1,00 \\
\hline Sim & $1,33(1,11 ; 1,59)$ & $1,22(1,03 ; 1,45)$ & $1,26(1,12 ; 1,42)$ & $1,16(1,04 ; 1,29)$ \\
\hline Doença cardiovascular & $p=0,030$ & $\mathrm{p}=0,073$ & $\mathrm{p}=0,008$ & $\mathrm{p}=0,193$ \\
\hline Não & 1,00 & 1,00 & 1,00 & 1,00 \\
\hline Sim & $1,20(1,02 ; 1,42)$ & $1,16(0,99 ; 1,36)$ & $1,16(1,04 ; 1,29)$ & $1,07(0,96 ; 1,20)$ \\
\hline Hipercolesterolemia & $\mathrm{p}=0,585$ & - & $\mathrm{p}=0,001$ & $\mathrm{p}=0,874$ \\
\hline Não & 1,00 & - & 1,00 & 1,00 \\
\hline $\operatorname{Sim}$ & $1,05(0,89 ; 1,24)$ & - & $1,18(1,07 ; 1,30)$ & $1,01(0,92 ; 1,11)$ \\
\hline
\end{tabular}

*Teste qui-quadrado para tendência linear.

valência $2 \%$ menor, comparando-se aos idosos de 60 a 69 anos, e aqueles com 80 anos ou mais apresentaram uma prevalência $27 \%$ menor, ao comparar-se com a categoria de referência. Sobre as variáveis comportamentais, os fumantes apresentaram uma prevalência $21 \%$ menor, quando comparados aos não fumantes, e aqueles idosos fisicamente ativos mostraram prevalência $26 \%$ 
Tabela 3. Análises bruta e ajustada entre obesidade geral e variáveis independentes estratificadas por sexo. Pelotas, RS, Brasil, 2014.

\begin{tabular}{|c|c|c|c|c|}
\hline \multirow[b]{2}{*}{ Variáveis } & \multicolumn{2}{|c|}{ Homens } & \multicolumn{2}{|c|}{ Mulheres } \\
\hline & $\begin{array}{c}\text { Bruta } \\
\text { RP (IC95\%) }\end{array}$ & $\begin{array}{c}\text { Ajustada } \\
\text { RP (IC95\%) }\end{array}$ & $\begin{array}{c}\text { Bruta } \\
\text { RP (IC95\%) }\end{array}$ & $\begin{array}{c}\text { Ajustada } \\
\text { RP (IC95\%) }\end{array}$ \\
\hline \multicolumn{5}{|l|}{ 1 Nível } \\
\hline Idade (anos) & $\mathrm{p}=0,001^{*}$ & $\mathrm{p}=0,001^{*}$ & $\mathrm{p}<0,001^{*}$ & $\mathrm{p}<0,001^{*}$ \\
\hline $60-69$ & 1,00 & 1,00 & 1,00 & 1,00 \\
\hline $70-79$ & $0,65(0,47 ; 0,90)$ & $0,64(0,46 ; 0,89)$ & $0,88(0,72 ; 1,09)$ & $0,79(0,64 ; 0,97)$ \\
\hline $80+$ & $0,48(0,28 ; 0,84)$ & $0,46(0,26 ; 0,81)$ & $0,55(0,40 ; 0,77)$ & $0,48(0,34 ; 0,68)$ \\
\hline Cor da pele & $p=0,676$ & - & $\mathrm{p}=0,280$ & - \\
\hline Branca & 1,00 & - & 1,00 & - \\
\hline Preta/Parda/Amarela/Indígena & $1,09(0,74 ; 1,59)$ & - & $1,15(0,89 ; 1,48)$ & - \\
\hline Escolaridade & $\mathrm{p}=0,048^{\star}$ & $\mathrm{p}=0,420$ & $\mathrm{p}=0,235$ & - \\
\hline $0-3$ & 1,00 & 1,00 & 1,00 & - \\
\hline $4-7$ & $1,15(0,81 ; 1,63)$ & $1,08(0,77 ; 1,54)$ & $1,21(0,97 ; 1,50)$ & - \\
\hline $8-11$ & $1,22(0,71 ; 2,11)$ & $1,13(0,65 ; 1,97)$ & $1,03(0,73 ; 1,45)$ & - \\
\hline $12+$ & $1,61(1,02 ; 2,53)$ & $1,47(0,92 ; 2,33)$ & $0,95(0,74 ; 1,21)$ & - \\
\hline Classe econômica (ABEP) & $\mathrm{p}=0,211$ & - & $\mathrm{p}=0,039$ & $\mathrm{p}=0,056$ \\
\hline $\mathrm{A} / \mathrm{B}$ (mais ricos) & 1,00 & - & 1,00 & 1,00 \\
\hline $\mathrm{C}$ & $0,73(0,51 ; 1,04)$ & - & $1,14(0,92 ; 1,41)$ & $1,14(0,92 ; 1,41)$ \\
\hline D/E (mais pobres) & $0,81(0,47 ; 1,39)$ & - & $0,73(0,48 ; 1,10)$ & $0,76(0,50 ; 1,14)$ \\
\hline Situação conjugal & $\mathrm{p}=0,449$ & - & $\mathrm{p}=0,028$ & $\mathrm{p}=0,359$ \\
\hline Com companheiro (a) & 1,00 & - & 1,00 & 1,00 \\
\hline Sem companheiro (a) & $0,85(0,55 ; 1,30)$ & - & $0,82(0,69 ; 0,98)$ & $0,92(0,76 ; 1,11)$ \\
\hline \multicolumn{5}{|l|}{ 2o Nível } \\
\hline Tabagismo atual & $\mathrm{p}=0,639$ & - & $\mathrm{p}=0,049$ & $\mathrm{p}=0,020$ \\
\hline Não & 1,00 & - & 1,00 & 1,00 \\
\hline Sim & $0,88(0,51 ; 1,51)$ & - & $0,68(0,46 ; 1,00)$ & $0,64(0,44 ; 0,93)$ \\
\hline Atividade física (lazer) & $\mathrm{p}=0,483$ & - & $\mathrm{p}=0,067$ & $\mathrm{p}=0,022$ \\
\hline Insuficientemente ativo & 1,00 & - & 1,00 & 1,00 \\
\hline Ativo & $0,86(0,57 ; 1,31)$ & - & $0,74(0,53 ; 1,02)$ & $0,69(0,50 ; 0,95)$ \\
\hline \multicolumn{5}{|l|}{ 3o Nível } \\
\hline Hipertensão arterial & $\mathrm{p}=0,045$ & $\mathrm{p}=0,027$ & $\mathrm{p}=0,005$ & $\mathrm{p}=0,026$ \\
\hline Não & 1,00 & 1,00 & 1,00 & 1,00 \\
\hline $\operatorname{Sim}$ & $1,41(1,01 ; 1,97)$ & $1,46(1,05 ; 2,05)$ & $1,49(1,13 ; 1,95)$ & $1,36(1,04 ; 1,78)$ \\
\hline Diabetes & $\mathrm{p}=0,052$ & $\mathrm{p}=0,073$ & $\mathrm{p}=0,015$ & $\mathrm{p}=0,255$ \\
\hline Não & 1,00 & 1,00 & 1,00 & 1,00 \\
\hline Sim & $1,39(1,00 ; 1,92)$ & $1,34(0,97 ; 1,86)$ & $1,31(1,06 ; 1,64)$ & $1,13(0,92 ; 1,39)$ \\
\hline Doença cardiovascular & $\mathrm{p}=0,825$ & - & $\mathrm{p}=0,015$ & $\mathrm{p}=0,018$ \\
\hline Não & 1,00 & - & 1,00 & 1,00 \\
\hline $\operatorname{Sim}$ & $1,04(0,75 ; 1,43)$ & - & $1,29(1,05 ; 1,57)$ & $1,26(1,04 ; 1,52)$ \\
\hline Hipercolesterolemia & $\mathrm{p}=0,492$ & - & $\mathrm{p}=0,803$ & - \\
\hline Não & 1,00 & - & 1,00 & - \\
\hline $\operatorname{Sim}$ & $1,10(0,83 ; 1,47)$ & - & $0,98(0,80 ; 1,19)$ & - \\
\hline
\end{tabular}

*Teste qui-quadrado para tendência linear.

menor, comparando-se aos insuficientemente ativos. Entre os idosos que referiram ser hipertensos e diabéticos foram observadas prevalências $44 \%$ e $16 \%$ maiores de obesidade abdominal, respectivamente, quando comparados àqueles que não referiram tais doenças (Tabela 4).

\section{Discussão}

A prevalência de obesidade geral entre idosos deste estudo foi cerca de $30 \%$. Esse resultado é superior ao encontrado na Pesquisa Nacional de Saúde e Nutrição (PNSN) de 1989, que foi de 
Tabela 4. Análises bruta e ajustada entre obesidade abdominal e variáveis independentes estratificadas por sexo. Pelotas, RS, Brasil, 2014.

\begin{tabular}{|c|c|c|c|c|}
\hline \multirow[b]{2}{*}{ Variáveis } & \multicolumn{2}{|c|}{ Homens } & \multicolumn{2}{|c|}{ Mulheres } \\
\hline & $\begin{array}{c}\text { Bruta } \\
\text { RP (IC95\%) }\end{array}$ & $\begin{array}{c}\text { Ajustada } \\
\text { RP }(\text { IC95\%) }\end{array}$ & $\begin{array}{c}\text { Bruta } \\
\text { RP }(\text { IC95\%) }\end{array}$ & $\begin{array}{c}\text { Ajustada } \\
\text { RP (IC95\%) }\end{array}$ \\
\hline \multicolumn{5}{|l|}{$1^{\circ}$ Nível } \\
\hline Idade (anos) & $\mathrm{p}=0,173$ & $\mathrm{p}=0,173$ & $\mathrm{p}=0,003$ & $p=0,006$ \\
\hline $60-69$ & 1,00 & 1,00 & 1,00 & 1,00 \\
\hline $70-79$ & $0,82(0,63 ; 1,07)$ & $0,82(0,63 ; 1,07)$ & $1,13(1,01 ; 1,26)$ & $1,07(0,96 ; 1,19)$ \\
\hline $80+$ & $0,72(0,47 ; 1,10)$ & $0,72(0,47 ; 1,10)$ & $0,83(0,70 ; 0,97)$ & $0,78(0,66 ; 0,94)$ \\
\hline Cor da pele & $\mathrm{p}=0,419$ & - & $\mathrm{p}=0,261$ & - \\
\hline Branca & 1,00 & - & 1,00 & - \\
\hline Preta/Parda/Amarela/Indígena & $0,85(0,58 ; 1,26)$ & - & $0,91(0,78 ; 1,07)$ & - \\
\hline Escolaridade & $\mathrm{p}=0,683$ & - & $\mathrm{p}=0,044^{*}$ & $\mathrm{p}=0,300$ \\
\hline $0-3$ & 1,00 & - & 1,00 & 1,00 \\
\hline $4-7$ & $0,98(0,72 ; 1,33)$ & - & $1,10(0,97 ; 1,24)$ & $1,08(0,96 ; 1,22)$ \\
\hline $8-11$ & $1,22(0,81 ; 1,85)$ & - & $0,95(0,79 ; 1,14)$ & $0,95(0,79 ; 1,15)$ \\
\hline $12+$ & $1,15(0,81 ; 1,62)$ & - & $0,88(0,76 ; 1,02)$ & $0,96(0,82 ; 1,11)$ \\
\hline Classe econômica (ABEP) & $\mathrm{p}=0,038^{\star}$ & $\mathrm{p}=0,172$ & $\mathrm{p}=0,051$ & $\mathrm{p}=0,202$ \\
\hline $\mathrm{A} / \mathrm{B}$ (mais ricos) & 1,00 & 1,00 & 1,00 & 1,00 \\
\hline $\mathrm{C}$ & $0,78(0,60 ; 1,02)$ & $0,80(0,60 ; 1,05)$ & $1,16(1,01 ; 1,32)$ & $1,08(0,92 ; 1,26)$ \\
\hline $\mathrm{D} / \mathrm{E}$ (mais pobres) & $0,64(0,39 ; 1,06)$ & $0,67(0,40 ; 1,11)$ & $0,99(0,79 ; 1,22)$ & $0,91(0,72 ; 1,17)$ \\
\hline Situação conjugal & $\mathrm{p}=0,205$ & - & $\mathrm{p}=0,786$ & - \\
\hline Com companheiro (a) & 1,00 & - & 1,00 & - \\
\hline Sem companheiro (a) & $0,80(0,57 ; 1,13)$ & - & $0,99(0,88 ; 1,10)$ & - \\
\hline \multicolumn{5}{|l|}{ 2 Nível } \\
\hline Tabagismo atual & $\mathrm{p}=0,150$ & $\mathrm{p}=0,114$ & $\mathrm{p}=0,049$ & $\mathrm{p}=0,109$ \\
\hline Não & 1,00 & 1,00 & 1,00 & 1,00 \\
\hline Sim & $0,72(0,46 ; 1,13)$ & $0,70(0,45 ; 1,09)$ & $0,81(0,66 ; 1,00)$ & $0,84(0,68 ; 1,04)$ \\
\hline Atividade física (lazer) & $\mathrm{p}=0,045$ & $\mathrm{p}=0,060$ & $\mathrm{p}=0,012$ & $\mathrm{p}=0,016$ \\
\hline Insuficientemente ativo & 1,00 & 1,00 & 1,00 & 1,00 \\
\hline Ativo & $0,67(0,46 ; 0,99)$ & $0,70(0,48 ; 1,02)$ & $0,79(0,66 ; 0,95)$ & $0,80(0,66 ; 0,96)$ \\
\hline \multicolumn{5}{|l|}{ 3o Nível } \\
\hline Hipertensão arterial & $\mathrm{p}<0,001$ & $\mathrm{p}<0,001$ & $\mathrm{p}<0,001$ & $\mathrm{p}=0,001$ \\
\hline Não & 1,00 & 1,00 & 1,00 & 1,00 \\
\hline Sim & $1,93(1,41 ; 2,66)$ & $1,93(1,41 ; 2,66)$ & $1,40(1,19 ; 1,64)$ & $1,32(1,12 ; 1,55)$ \\
\hline Diabetes & $\mathrm{p}=0,041$ & $\mathrm{p}=0,103$ & $\mathrm{p}<0,001$ & $\mathrm{p}=0,030$ \\
\hline Não & 1,00 & 1,00 & 1,00 & 1,00 \\
\hline Sim & $1,34(1,01 ; 1,76)$ & $1,26(0,95 ; 1,66)$ & $1,26(1,12 ; 1,41)$ & $1,14(1,01 ; 1,29)$ \\
\hline Doença cardiovascular & $\mathrm{p}=0,250$ & - & $\mathrm{p}=0,003$ & $\mathrm{p}=0,147$ \\
\hline Não & 1,00 & - & 1,00 & 1,00 \\
\hline Sim & $1,16(0,90 ; 1,49)$ & - & $1,18(1,06 ; 1,31)$ & $1,09(0,97 ; 1,22)$ \\
\hline Hipercolesterolemia & $\mathrm{p}=0,086$ & $\mathrm{p}=0,552$ & $\mathrm{p}=0,233$ & - \\
\hline Não & 1,00 & 1,00 & 1,00 & - \\
\hline Sim & $1,27(0,97 ; 1,67)$ & $1,09(0,82 ; 1,44)$ & $1,06(0,96 ; 1,16)$ & - \\
\hline
\end{tabular}

* Teste qui-quadrado para tendência linear.

$17 \%{ }^{23}$, e aos $17 \%$ observados na Pesquisa de Orçamentos Familiares (POF) de 2008-2009, entre indivíduos de 65 anos ou mais ${ }^{6}$. Em Pelotas, RS, no ano 2000, em estudo também de base populacional, a prevalência encontrada foi de $25 \%{ }^{24}$. As diferenças, no caso da PNSN e da POF, podem ser explicadas em parte pelo tempo decor- rido desde a sua realização, representando uma evolução da prevalência, e porque representam o Brasil como um todo, diferente deste estudo, que se refere a apenas a área urbana de uma cidade de porte médio onde, sabidamente, a prevalência de obesidade é maior que no país como um todo. Em relação ao estudo de Pelotas, além do tempo 
decorrido, a diferença pode ter sido devido à forma de obtenção das medidas de peso e altura, que foram autorreferidas. Destaca-se ainda o fato de, neste estudo, a altura ter sido estimada a partir da medida da altura do joelho.

Metade dos idosos estudados apresentou obesidade abdominal. Um estudo realizado em 2010 com uma amostra representativa da população adulta de Pelotas (RS) encontrou que aproximadamente $46 \%$ dos indivíduos no estrato de 60 anos ou mais apresentava obesidade abdominal $^{13}$. Outro estudo de base populacional, conduzido em 1996 no município de Rio de Janeiro, encontrou uma prevalência de inadequação do perímetro da cintura superior a $50 \%$, porém, utilizando pontos de corte $\geq 94 \mathrm{~cm}$, para homens, e $\geq 80 \mathrm{~cm}$, para mulheres ${ }^{17}$. A prevalência do presente estudo pode ser considerada maior que a do estudo supracitado, em virtude de nossos pontos de corte serem mais conservadores. Em comparação ao estudo de Linhares et al. ${ }^{13}$, que utilizou os mesmos pontos de corte, nossa prevalência foi também superior, sugerindo um possível aumento da obesidade abdominal entre idosos pelotenses no período de 2010 a 2014 .

Alguns autores têm discutido que as modificações decorrentes do processo de envelhecimento podem fazer com que o IMC não reflita adequadamente a adiposidade corporal. Batsis et al. ${ }^{25}$, analisando uma coorte representativa de idosos dos Estados Unidos, e Choi et al. ${ }^{26}$ uma coorte de idosos coreanos, observaram que indivíduos com IMC normal, mas obesos metabolicamente, ou seja, com gordura corporal elevada, apresentavam alto risco de desregulação cardiometabólica e mortalidade. Apesar disso, estudos encontram boa correlação entre circunferência da cintura e $\mathrm{IMC}^{17,27}$, podendo este ser útil para o diagnóstico de obesidade em nível populacional ${ }^{4}$. No presente estudo, a maioria das variáveis que se mantiveram relacionadas à obesidade geral e à obesidade abdominal, após a análise ajustada, apresentou direções de associação semelhantes, exceto para a variável idade.

Com relação ao sexo, encontrou-se maior prevalência de obesidade, principalmente abdominal, em mulheres. Barreto et al. ${ }^{28}$, ao estudar uma coorte de idosos de Bambuí, MG, observou que o sexo feminino foi positivamente associado ao IMC. Santos e Sichieri ${ }^{17}$ relataram o mesmo para obesidade abdominal, sendo que as idosas do Rio de Janeiro apresentaram maior proporção de inadequação da circunferência da cintura. Dois fatores podem explicar estes resultados. Primeiramente, o acúmulo de gordura subcutânea durante o processo de envelhecimento é maior em mulheres do que em homens e a perda de gordura se dá em idade mais avançada em idosas $^{14}$. Além disso, devido à expectativa de vida ser maior entre as mulheres, pode ter ocorrido viés de sobrevivência, contribuindo para subestimação da prevalência de obesidade em homens.

Os resultados aqui apresentados mostram que a idade esteve negativamente associada à obesidade geral e abdominal, observando-se tendência linear de declínio com o avanço das faixas etárias. Tais resultados poderiam ser explicados pelas alterações que ocorrem no processo de envelhecimento. A diminuição de peso nos idosos, inerente a problemas alimentares, como perda de apetite e dificuldade de mastigação por lesão oral, uso de prótese dentária ou problemas digestivos, leva a uma redução do $\mathrm{IMC}^{2}$. Ao mesmo tempo, há uma redução da massa muscular devido à sua transformação em gordura intramuscular, também contribuindo para a diminuição do peso ${ }^{8}$. Ainda, a categoria de 80 anos ou mais de idade mostrou as menores prevalências de obesidade geral e abdominal, podendo ser explicado pelo viés de sobrevivência, que diz respeito à mortalidade em indivíduos com doenças relacionadas à obesidade.

Quanto às características comportamentais dos idosos, encontrou-se que indivíduos fumantes apresentaram menores prevalências, tanto de obesidade geral como abdominal. Os mecanismos da perda de peso associada ao hábito de fumar são complexos e refletem os efeitos mediados pela nicotina. Essa substância reduz o peso corporal a partir do aumento da taxa de metabolismo basal, além de regular os múltiplos caminhos neuroquímicos que governam a fome e a saciedade ${ }^{29}$. Em relação à atividade física, foi observado que as prevalências de obesidade geral e abdominal eram menores em idosos fisicamente ativos do que naqueles insuficientemente. Em decorrência do declínio funcional natural ao envelhecimento, bem como à carga de doenças presente nesta faixa etária, há um aumento nas limitações físicas, dificultando a prática de atividades físicas, com consequente aumento da obesidade $^{30}$. Entretanto, deve-se ter cautela na interpretação das relações entre tabagismo, atividade física e obesidade devido à presença de causalidade reversa.

Os resultados deste estudo mostram que a obesidade esteve positivamente associada à HAS e à DM, corroborando os achados de Barreto et al. nos idosos da coorte de Bambuí, $\mathrm{MG}^{28}$. Entretanto, no presente estudo, não é possível inferir que a 
obesidade representa uma causa de tais doenças, mas, apenas, que ela está presente em hipertensos e diabéticos. Sabe-se que a obesidade é um agravo metabólico complexo, que envolve redução da sensibilidade à insulina e consequente estado de descompensação nos níveis de glicose circulante, bem como perfis lipídicos adversos. Ainda, as alterações de perfil metabólico mostram-se mais comuns naqueles indivíduos com acúmulo de gordura intraabdominal e representam fatores de risco para DM e doenças cardiovasculares ${ }^{10,31}$.

Algumas variáveis demográficas (cor da pele e situação conjugal) e socioeconômicas (escolaridade e classe econômica) não se mostraram associadas à obesidade geral e à abdominal. Silveira et al., estudando idosos em Pelotas, RS, mostraram que renda per capita, escolaridade e cor da pele não tiveram associação com obesidade geral ${ }^{24}$. Ressalta-se que a obesidade pode estar difundida em tamanha escala na sociedade que, em idosos, as questões inerentes ao processo de envelhecimento mostram maior efeito. Com relação à doença cardiovascular e à hipercolesterolemia, variáveis que perderam a associação com obesidade geral e abdominal após o ajuste, poderiam estar sob efeito de confusão na análise bruta por parte da HAS.

Como pontos fortes do estudo, é importante ressaltar que o mesmo foi de caráter populacional, delineado especificamente para indivíduos idosos. Além disso, a coleta de medidas antropométricas, ao invés de peso e altura autorreferidos, confere confiabilidade ao estudo. Alguns estudos foram realizados no Brasil para determinar a validade dessas medidas autorreferidas em indivíduos de 20 anos ou mais ${ }^{32,33}$ e 12 anos ou mais ${ }^{34}$ e os autores mostram que a idade afeta a qualidade do IMC referido ${ }^{32,33}$ e que os idosos tendem a superestimar a altura ${ }^{34}$, possivelmente por não verificarem suas medidas periodicamente e relatarem informações imprecisas, baseadas em recordatório de medidas passadas ${ }^{33}$.

A utilização da medida de altura do joelho, em substituição à altura em pé, está de acordo com o preconizado pela OMS para a avaliação antropométrica da população idosa ${ }^{14}$, considerando que o declínio da estatura, decorrente de compressão vertebral e perda de tônus muscular, está presente no processo de envelhecimento ${ }^{2}$. Um estudo de revisão mostrou que as equações desenvolvidas por Chumlea e $\mathrm{Guo}^{15}$, utilizadas no presente estudo, são as que possuem maior número de trabalhos avaliando sua aplicação, e que sabe-se que há possibilidade de superestimar a altura ao utilizá-las na população brasileira ${ }^{35}$. Por esse motivo, na tentativa de garantir a qualidade desta medida, ava- liou-se a altura em pé de uma subamostra de idosos de Pelotas-RS sem curvatura na coluna e o cálculo do coeficiente de concordância de Lin entre esta medida e a altura do joelho resultou em alta concordância entre as duas medidas $(r=0,868)$. A média de altura real foi de $166,92 \mathrm{~cm}(\mathrm{DP}=5,50$ $\mathrm{cm})$ para homens e de $155,18 \mathrm{~cm}(\mathrm{DP}=7,34 \mathrm{~cm})$ para mulheres e a média de altura estimada foi de $167,86 \mathrm{~cm}(\mathrm{DP}=4,84 \mathrm{~cm})$ para homens e de $156,41 \mathrm{~cm}(\mathrm{DP}=5,16 \mathrm{~cm})$ para mulheres.

Algumas limitações devem ser consideradas. A diferença encontrada entre as médias de idade de perdas e recusas, em comparação à amostra estudada (69,5 anos; DP $\pm 8,6$ vs. 70,7; DP $\pm 8,2$, respectivamente), poderia estar subestimando a prevalência de obesidade geral e abdominal na amostra, já que idosos mais novos apresentaram maior prevalência. Entretanto, tal fato provavelmente não é relevante, pois a diferença entre as médias foi muito pequena $(1,2 ; \mathrm{DP} \pm 0,47)$.

As informações coletadas sobre morbidade foram provenientes de autorrelato do entrevistado, uma estratégia que estima as prevalências com menor custo e de forma acessível e rápida, mas que pode apresentar erros de classificação. Contudo, tais questões foram construídas com base em um estudo realizado com 2.949 indivíduos de Pelotas (RS) que mostrou ser válido o autorrelato de $\mathrm{HAS}^{36}$, sendo, então, extrapolado para as outras doenças. Outra limitação refere-se ao viés de causalidade reversa, inerente aos estudos transversais, nos quais não é possível estabelecer uma relação de temporalidade entre determinadas variáveis independentes e os desfechos, como nas associações de tabagismo, atividade física e morbidade autorreferida com obesidade geral e abdominal.

No presente estudo, foi utilizado como ponto de corte para obesidade IMC $\geq 30 \mathrm{~kg} / \mathrm{m}^{2}$, proposto para adultos pela $\mathrm{OMS}^{14}$. A definição da OMS, assim como a da Organização Pan-Americana da Saúde ${ }^{37}$, permite discriminar a obesidade do excesso de peso, ainda que não possua distinção para os diferentes grupos etários. Considerando as alterações fisiológicas decorrentes do envelhecimento, o Ministério da Saúde ${ }^{16}$ recomenda a proposta de Lipschitz ${ }^{38}$, que define o IMC $>27 \mathrm{~kg} /$ $\mathrm{m}^{2}$ como causa de preocupação em idosos. Esse autor, ainda, discute que é geralmente recomendado que idosos tenham um IMC entre 24 e 29 $\mathrm{kg} / \mathrm{m}^{2}$, limites aceitáveis semelhantes à definição de obesidade da OMS e da OPAS. Devido à ausência de consenso sobre o ponto de corte mais adequado na definição de obesidade em idosos, utilizou-se a recomendação da OMS no presente 
estudo, por essa estar mais consolidada na literatura sobre o tema. A partir de revisão da literatura, observa-se que a maioria dos estudos, nacionais e internacionais, utiliza essa classificação ${ }^{39-43}$ e poucos estudos brasileiros utilizam a recomendação do Ministério da Saúde ${ }^{44,45}$. Ainda, foi encontrado um estudo que comparou os dois pontos de cor$t^{24}$. Caso o ponto de corte para excesso de peso $\left(\right.$ IMC $\left.>27 \mathrm{~kg} / \mathrm{m}^{2}\right)$ viesse a ser utilizado, a prevalência na população estudada seria $56 \%$.

Através deste estudo transversal de base populacional foi possível estimar as prevalências e identificar os fatores associados à obesidade geral e abdominal em idosos. A elucidação de como a obesidade se distribui na população em estudo fornece subsídios para o planejamento de políticas de saúde que visem à redução da sua ocorrência. No entanto, é necessário compreender que as alterações fisiológicas inerentes ao processo de envelhecimento não podem ser evitadas, mas podem ser atenuadas a partir de ações que englobem o incentivo à prática de atividade física, por exemplo, considerando as limitações e condições de saúde do idoso.

\section{Colaboradores}

CS Costa participou de todas as fases do estudo, desde a concepção e planejamento, análise e interpretação dos dados, redação do artigo e aprovação da versão final do manuscrito; BC Schneider e JA Cesar contribuíram substancialmente para a concepção e planejamento, interpretação dos dados e revisão crítica do conteúdo e aprovaram a versão final do manuscrito.

\section{Referências}

1. World Health Organization (WHO). Definition of an older or elderly person. [acessado 2013 abr 4]. Disponível em: http://www.who.int/healthinfo/survey/age ingdefnolder/en/.

2. Brasil. Ministério da Saúde (MS). Secretaria de Atenção à Saúde. Departamento de Atenção Básica. Envelhecimento e saúde da pessoa idosa. Brasília: MS; 2007.

3. Mazzoccante RP, Moraes JFVN, Campbell CSG. Gastos públicos diretos com a obesidade e doenças associadas no Brasil. Rev. Ciênc. Méd. 2013; 21(1-6):25-34.

4. Brasil. Ministério da Saúde (MS). Secretaria de Atenção à Saúde. Departamento de Atenção Básica. Obesidade. Brasília: MS; 2006.

5. Finucane MM, Stevens GA, Cowan MJ, Danaei G, Lin JK, Paciorek CJ, Singh GM, Gutierrez HR, Lu Y, Bahalim AN, Farzadfar F, Riley LM, Ezzati M; Global Burden of Metabolic Risk Factors of Chronic Diseases Collaborating Group (Body Mass Index). National, regional, and global trends in body-mass index since 1980: systematic analysis of health examination surveys and epidemiological studies with 960 country-years and 9.1 million participants. Lancet 2011; 377(9765):557-567.

6. Instituto Brasileiro de Geografia e Estatística (IBGE) Pesquisa de Orçamentos Familiares 2008-2009. Antropometria e estado nutricional de crianças, adolescentes e adultos no Brasil. Rio de Janeiro: 2010.

7. Fernihough A, McGovern ME. Physical stature decline and the health status of the elderly population in England. Econ Hum Biol 2015; 16:30-44.

8. Beaufrère $B$, Morio $B$. Fat and protein redistribution with aging: metabolic considerations. Eur J Clin Nutr 2000; 54(Supl. 3):S48-S53.
9. World Health Organization (WHO). Obesity and overweight. [acessado 2014 out 22]. Disponível em: http:// www.who.int/mediacentre/factsheets/fs311/en/.

10. World Health Organization (WHO). Waist circumference and waist-hip ratio: report of a WHO expert consultation. Geneva: WHO; 2008.

11. Instituto Brasileiro de Geografia e Estatística (IBGE). Censo Demográfico 2010: Características da População e dos Domicílios - Resultados do Universo. Rio de Janeiro: IBGE; 2011

12. Barros AJ, Menezes AMB, Santos IS, Assunção MCF, Gigante D, Fassa AG, Marques M, Araújo C, Hallal PC, Facchini LA. O Mestrado do Programa de Pós-graduacão em Epidemiologia da UFPel baseado em consórcio de pesquisa: uma experiência inovadora. Rev. bras. epidemiol. 2008; 11(Supl. 1):133-144.

13. Linhares RS, Horta BL, Gigante DP, Dias-da-Costa JS, Olinto MTA. Distribuição de obesidade geral e abdominal em adultos de uma cidade no Sul do Brasil. Cad Saude Publica 2012; 28(3):438-447.

14. World Health Organization (WHO). Physical status: The use and interpretation of anthropometry. Geneva: WHO; 1995. Report of a WHO expert committee. WHO technical report series. 854

15. Chumlea WC, Guo S. Equations for predicting stature in white and black elderly individuals. J Gerontol 1992; 47(6):M197-M203.

16. Brasil. Ministério da Saúde (MS). Vigilância alimentar e nutricional - Sisvan: orientações básicas para a coleta, processamento, análise de dados e informação em serviços de saúde. Brasília: MS; 2004. 
17. Santos DM, Sichieri R. Índice de massa corporal e indicadores antropométricos de adiposidade em idosos. Rev Saude Publica 2005; 39(2):163-168.

18. Olinto MTA, Nácul LC, Dias-da-Costa JS, Gigante DP, Menezes AMB, Macedo S. Níveis de intervenção para obesidade abdominal: prevalência e fatores associados. Cad Saude Publica 2006; 22(6):1207-1215.

19. Associação Brasileira de Empresas de Pesquisa (ABEP). Critério de classificação econômica Brasil. São Paulo: ABEP; 2014

20. Craig CL, Marshall AL, Sjöström M, Bauman AE, Booth ML, Ainsworth BE, Ainsworth BE, Pratt M, Ekelund U, Yngve A, Sallis JF, Oja P. International physical activity questionnaire: 12-country reliability and validity. Med. sci. sports exerc. 2003; 35(8):1381-1395.

21. Habicht J. Estandarización de métodos epidemiológicos cuantitativos sobre el terreno. Bol. Oficina Sanit. Panam. 1974; 76(5):375-384.

22. Victora CG, Huttly SR, Fuchs SC, Olinto MTA. The role of conceptual frameworks in epidemiological analysis: a hierarchical approach. Int. j. epidemiol. 1997; 26(1):224-227.

23. Tavares EL, Anjos LA. Perfil antropométrico da população idosa brasileira. Resultados da Pesquisa Nacional sobre Saúde e Nutrição. Cad Saude Publica 1999; 15(4):759-768.

24. Silveira EA, Kac G, Barbosa LS. Prevalência e fatores associados à obesidade em idosos residentes em Pelotas, Rio Grande do Sul, Brasil: classificação da obesidade segundo dois pontos de corte do índice de massa corporal. Cad Saude Publica 2009; 25(7):1569-1577.

25. Batsis JA, Sahakyan KR, Rodriguez-Escudero JP, Bartels SJ, Somers VK, Lopez-Jimenez F. Normal Weight Obesity and Mortality in United States Subjects $\geq 60$ Years of Age (from the Third National Health and Nutrition Examination Survey). Am. j. cardiol. 2013; 112(10):1592-1598.

26. Choi KM, Cho HJ, Choi HY, Yang SJ, Yoo HJ, Seo JA, Kim SG, Baik SH, Choi DS, Kim NH. Higher mortality in metabolically obese normal-weight people than in metabolically healthy obese subjects in elderly Koreans. Clin. endocrinol. 2013; 79(3):364-370.

27. Oka R, Miura K, Sakurai M, Nakamura K, Yagi K, Miyamoto S, Moriuchi T, Mabuchi H, Yamagishi M, Takeda Y, Hifumi S, Inazu A, Nohara A, Kawashiri MA, Kobayashi J. Comparison of waist circumference with body mass index for predicting abdominal adipose tissue. Diabetes res. clin. pract. 2009; 83(1):100-105.

28. Barreto SM, Passos V, Lima-Costa MFF. Obesity and underweight among Brazilian elderly: the Bambuí Health and Aging Study. Cad Saude Publica 2003; 19(2):605-612.

29. Audrain-McGovern J, Benowitz N. Cigarette smoking, nicotine, and body weight. Clin. pharmacol. ther. 2011; 90(1):164-168.

30. World Health Organization (WHO). Envelhecimento ativo: uma política de saúde. Brasília: Organização Pan-Americana da Saúde; 2005.

31. Carvalho KMB. Obesidade. In: Carvalho KMB. Guia de nutrição: nutrição clínica no adulto. Barueri: Manole; 2005. p. 149-170.

32. Silveira EA, Araújo CL, Gigante DP, Barros AJD, Lima MS. Validação do peso e altura referidos para o diagnóstico do estado nutricional em uma população de adultos no Sul do Brasil. Cad Saude Publica 2005; 21(1):235-245.
33. Del Duca, GF, González-Chica DA, Santos JV, Knuth AG, Camargo MBJ, Araújo CL. Peso e altura autorreferidos para determinação do estado nutricional de adultos e idosos: validade e implicações em análises de dados. Cad Saude Publica 2012; 28(1):75-85.

34. Carvalho AM, Piovezan LG, Selem SSC, Fisberg RM, Marchioni DML. Validação e calibração de medidas de peso e altura autorreferidas por indivíduos da cidade de São Paulo. Rev. bras. epidemiol. 2014; 17(3):735-746.

35. Monteiro RSC, Cunha TRL, Santos MEN, Mendonça SS. Estimativa de peso, altura e índice de massa corporal em adultos e idosos americanos: revisão. Com. Ciências Saúde 2009; 20(4):341-350

36. Chrestani MAD, Santos IS, Matijasevich AM. Hipertensão arterial sistêmica auto-referida: validação diagnóstica em estudo de base populacional. Cad Saude Publica 2009; 25(11):2395-2406.

37. Organização Pan-Americana da Saúde (OPAS). Encuesta multicentrica: salud, bien estar y envejecimiento (SABE) em América Latina y el Caribe. In: Anales da $36^{a}$ Reunión del Comité Asesor de Investigaciones en salud. Washington, D. C.: 2001.

38. Lipschitz DA. Screening for nutritional status in the elderly. Prim. care 1994; 21(1):55-67.

39. Suzana S, Kee CC, Jamaludin AR, Noor Safiza MN, Khor GL, Jamaiyah H, Geeta A, Ahmad Ali Z, Rahmah R, Ruzita AT, Ahmad Fauzi Y. The Third National Health and Morbidity Survey: prevalence of obesity, and abdominal obesity among the Malaysian elderly population. Asia Pac. j public health 2012; 24(2):318-329.

40. Gomez-Cabello A, Pedrero-Chamizo R, Olivares PR, Luzardo L, Juez-Bengoechea A, Mata E, Albers U, Aznar S, Villa G, Espino L, Gusi N, Gonzalez-Gross M, Casajus JA, Ara I on behalf of EXERNET Study Group. Prevalence of overweight and obesity in non-institutionalized people aged 65 or over from Spain: the elderly EXERNET multi-centre study. Obes. rev. 2011; 12(8):583-592.

41. Kvamme JM, Holmen J, Wilsgaard T, Florholmen J, Midthjell K, Jacobsen BK. Body mass index and mortality in elderly men and women: the Tromso and HUNT studies. J epidemiol. community health 2012; 66(7):611-617.

42. Bof AF, Caldas JAF, Kitoko PM, Batista JEM, Andrade TB. Prevalence of overweight and obesity in elderly people from Vitória-ES, Brazil. Cien Saude Colet 2012; 17(3):749-756.

43. Ferreira CCC, Peixoto MRG, Barbosa MA, Silveira EA. Prevalence of cardiovascular risk factors in elderly individuals treated in the Brazilian Public Health System in Goiania. Arq. bras. cardiol. 2010; 95(5):621-628.

44. Kümpel DA, Sodré AC, Pomatti DM, Scortegagna HM, Filippi J, Portella MR, Doring M, Scariot M. Obesidade em idosos acompanhados pela estratégia de saúde da família. Texto contexto-enferm. 2011; 20(3):471-477.

45. Scherer F, Vieira JLC. Estado nutricional e sua associação com risco cardiovascular e síndrome metabólica em idosos. Rev. nutr. 2010; 23(3):347-355.

Artigo apresentado em 26/09/2015

Aprovado em 04/05/2016

Versão final apresentada em 06/05/2016 